\title{
Generalized Quasi-Conformal Curvature Tensor On Normal Metric Contact Pairs
}

\author{
İnan Ünal \\ University of Munzur, Faculty of Engineering, 62000 Tunceli, Turkey \\ inanunal@munzur.edu.triD \\ Received date: 01.10.2020, Accepted date: 16.12.2020
}

\begin{abstract}
The aim of this paper is to examine normal metric contact pair (NMCP) manifolds under the flatness conditions on generalized quasi-conformal (GQC) curvature tensor. It is interested to classify GQC-flat and GQC-Z-flat NMCP manifolds. We prove that a GQC-flat NMCP manifold is a generalized quasi-Einstein (GQE) manifold and also, such manifolds are the space of generalized quasi-constant curvature. Finally, we consider the sectional curvature of NMCP manifolds under the flatness conditions of GQC curvature tensor.
\end{abstract}

Keywords: Contact metric pair, curvature properties, quasi-conformal curvature tensor

\section{Kontakt Metrik Çiftler Üzerinde Genelleştirilmiş Quasi-Conformal Eğrilik Tensörü}

\section{$\ddot{O ̈ z}$}

$\mathrm{Bu}$ çalışmanın amacı, normal metrik kontakt çift (NMCP) manifoldlarını genelleştirilmiş quasi-conformal eğrilik (GQC) tensörünün sıfirlık koşulları altında incelemektir. Bu kapsamda GQC-düz ve GQC-Z-düz NMCP manifoldları ile ilgilenilmiştir. Bir GQC-düz manifoldun genelleştirilmiş yarı-Einstein (GQE) manifold olduğu ve bu çeşit manifoldların genelleştirilmiş yarı-sabit eğriliğe sahip olduğu ispatlanmıştır. Son olarak, GQC eğrilik tensörünün düzlük şartları altında, NMCP manifoldlarının kesitsel eğrilikleri ele alınmıştır.

Anahtar Kelimeler: Kontakt metrik çift, eğrilik özellikleri, genelleştirilmiş quasi-conformal eğrilik tensörü

\section{INTRODUCTION}

Contact pair manifolds were firstly studied in (Blair et al., 1974). The authors worked on CalabiEckman manifold by considering complex manifolds. They obtained some results on contact and complex structures. Also, the authors presented the notion of bicontact manifolds. The issue of bicontact manifolds, which did not attract the attention of researchers for many years, began to be studied under the name of contact pairs in the 2000s. Bande and Hadjar, firstly gave the definition of a contact pair manifold (Bande and Hadjar, 2005). Then, they constructed an almost contact structure on a contact pair manifold and defined the associated metric (Bande and Hadjar, 2009). In 2013, the normality of almost contact metric pair structure were studied (Bande and Hadjar, 2013). Later, the normal contact metric pair (NMCP) manifolds have been studied by Bande, Hadjar and Blair in (Bande et al., 2010). In 2020, the presented author (Ünal,
2020) defined the notion of generalized quasiEinstein NMCP manifolds and obtained some results on curvature relations. Also, same author worked on certain flatness conditions on NMCP manifolds in (Ünal, 2020) and examined NMCP manifolds under semi-symmetry conditions (Ünal, 2020).

Curvature tensors are useful tools for to understand the global differential geometric properties of the manifolds with some special structures. Also, they give us some information about the global curvature properties of the manifolds, as they are associated with some special transformations. A type of curvature tensor was defined in (Baishya and Chowdhury, 2016) with the name of generalized quasi-conformal curvature tensor (GQC). A GQC curvature tensor $\mathrm{W}$ is defined on $\mathrm{k}$-dimensional Riemannian manifold as following; 


$$
\begin{aligned}
\mathrm{W}\left(\mathrm{Y}_{1}, \mathrm{Y}_{2}\right) \mathrm{Y}_{3} & =\mathrm{Rm}\left(\mathrm{Y}_{1}, \mathrm{Y}_{2}\right) \mathrm{Y}_{3} \\
& +\alpha\left[\sigma\left(\mathrm{Y}_{2}, \mathrm{Y}_{3}\right) \mathrm{Y}_{1}-\sigma\left(\mathrm{Y}_{1}, \mathrm{Y}_{3}\right) \mathrm{Y}_{2}\right] \\
& +\beta\left[\mathrm{g}\left(\mathrm{Y}_{2}, \mathrm{Y}_{3}\right) \mathrm{Q} \mathrm{Y}_{1}-\mathrm{g}\left(\mathrm{Y}_{1}, \mathrm{Y}_{3}\right) \mathrm{Q} \mathrm{Y}_{2}\right] \\
& -\frac{\gamma \mathrm{s}}{2 \mathrm{k}+1}\left(\frac{1}{2 \mathrm{k}}+\alpha+\beta\right)\left[\mathrm{g}\left(\mathrm{Y}_{2}, \mathrm{Y}_{3}\right) \mathrm{Y}_{1}\right. \\
& \left.-\mathrm{g}\left(\mathrm{Y}_{1}, \mathrm{Y}_{3}\right) \mathrm{Y}_{2}\right]
\end{aligned}
$$

for all $\mathrm{Y}_{1}, \mathrm{Y}_{2}, \mathrm{Y}_{3} \in \Gamma(\mathrm{TM})$, where $\mathrm{Rm}$ is the Riemannian curvature, $\sigma$ is Ricci curvature tensor, $\mathrm{Q}$ is the Ricci tensor, s is the scalar curvature and $\alpha, \beta, \gamma$ are constants. For the special values of $\alpha, \beta, \gamma$ we have same special curvature tensors as in Table 1.

A Riemannian manifold is said to be GQC flat if $\mathrm{W}=0$. The flatness of GQC curvature tensor also determines the flatness of some special curvature tensors mentioned Table1. GQC curvature tensor on some different manifolds with special structures have been studied in (Baishya and Chowdhury, 2017; Baishya 2017; Acet, 2018).

The aim of this paper is to examine normal metric contact pair (NMCP) manifolds under the flatness conditions on generalized quasi-conformal (GQC) curvature tensor. We classify GQC-flat and GQC-Z-flat NMCP manifolds.

Table 1. Some special curvature tensor

\begin{tabular}{cccc}
\hline$\alpha$ & $\beta$ & $\gamma$ & Curvature Tensor \\
\hline 0 & 0 & 0 & Riemann (Rm) \\
\hline$-\frac{1}{2 \mathrm{k}-1}$ & $-\frac{1}{2 \mathrm{k}-1}$ & 1 & Conformal (C) \\
\hline$-\frac{1}{2 \mathrm{k}-1}$ & $-\frac{1}{2 \mathrm{k}-1}$ & 0 & Conharmonic (K) \\
\hline 0 & 0 & 1 & Concircular ( $\tilde{\mathrm{C}})$ \\
\hline$-\frac{1}{2 \mathrm{k}}$ & 0 & 0 & Projective (P ) \\
\hline$-\frac{1}{4 \mathrm{k}}$ & $-\frac{1}{4 \mathrm{k}}$ & 0 & m-Projective (M ) \\
\hline
\end{tabular}

We prove that a GQC-flat manifold is a generalized quasi-Einstein (GQE) manifold and also, such manifolds are the space of generalized quasi-constant curvature. Finally, we consider the sectional curvature of NMCP manifolds under the flatness conditions of GQC curvature tensor.

\section{MATERIAL AND METHODS}

This chapter is avoided to review some of standard facts on the contact pairs. For more details, see (Bande and Hadjar, 2005; Bande and Hadjar, 2009; Blair et al. 2013).

Let $\mathrm{N}$ be a $\mathrm{k}$-dimensional differentiable manifold such that $k=2 m+2 n+2$ for positive $m, n$ integers. Two 1 -forms $\omega_{1}, \omega_{2}$ on $\mathrm{N}$ is called a contact pair of type $(\mathrm{m}, \mathrm{n})$ if we have

$$
\begin{aligned}
& \omega_{1} \wedge\left(d \omega_{1}\right)^{\mathrm{m}} \wedge \omega_{2} \wedge\left(d \omega_{2}\right)^{\mathrm{n}} \neq 0, \\
& \left(\mathrm{~d} \omega_{1}\right)^{\mathrm{m}+1}=0,\left(\mathrm{~d} \omega_{2}\right)^{\mathrm{n}+1}=0
\end{aligned}
$$

Then, we call $\mathrm{N}$ by a contact pair manifold (Bande and Hadjar, 2005).

We have two distributions $\mathrm{D}_{1}$ and $\mathrm{D}_{2}$ which are the kernels of $\omega_{1}$ and $\omega_{2}$, respectively. Also, two characteristic foliations of $\mathrm{N}$ are given by $\mathrm{F}_{1}=\mathrm{D}_{1} \cap \operatorname{kerd} \alpha_{1} \quad$ and $\quad \mathrm{F}_{2}=\mathrm{D}_{2} \cap \operatorname{kerd} \alpha_{2}$. Moreover, $F_{1}$ and $F_{2}$ has contact forms which are induced from $\omega_{2}, \omega_{1}$. The characteristic vector fields $Z_{1}$ and $Z_{2}$ of a contact pair manifold are given by $\alpha_{1}\left(Z_{1}\right)=\alpha_{2}\left(Z_{2}\right)=1, \alpha_{1}\left(Z_{2}\right)=\alpha_{2}\left(Z_{1}\right)=0$.

Two subbundles $\mathrm{TG}$ and $\mathrm{TG}_{2}$ are defined as $\mathrm{TG}=\operatorname{kerd} \alpha_{\mathrm{i}} \cap \operatorname{ker} \alpha_{1} \cap \operatorname{ker} \alpha_{2}$. Thus, we have

$\mathrm{TF}_{\mathrm{i}}=\mathrm{TG}_{\mathrm{i}} \oplus \mathbb{R} \mathrm{Z}_{\mathrm{j}}, 1 \leq \mathrm{i}, \mathrm{j} \leq 2, \mathrm{i} \neq \mathrm{j}$.

Finally, the decomposition of TN stated by

$\mathrm{TM}=\mathrm{TG}_{1} \oplus \mathrm{TG}_{2} \oplus \mathbb{R} \mathrm{Z}_{1} \oplus \mathbb{R} \mathrm{Z}_{2}, \mathrm{We}$ call the subbundle $\mathrm{H}=\mathrm{TG} \oplus \mathrm{TG}_{2}$ by horizontal subbundle and $\mathrm{V}=\mathbb{R} \mathrm{Z}_{1} \oplus \mathbb{R} \mathrm{Z}_{2}$ by vertical subbundle. Also, a vector field $Y_{1}$ is called as horizontal if it is in $\mathrm{H}$ and vertical if it is in $\mathrm{V}$.

A k-dimensional differentiable manifold $\mathrm{N}$ is called metric almost contact pair manifold if we have

$$
\phi^{2}=-\mathrm{I}+\alpha_{1} \otimes \mathrm{Z}_{1}+\alpha_{2} \otimes \mathrm{Z}_{2}, \quad \phi \mathrm{Z}_{1}=\phi \mathrm{Z}_{2}=0,
$$

$$
\begin{aligned}
\mathrm{g}\left(\phi \mathrm{Y}_{1}, \phi \mathrm{Y}_{2}\right) & =\mathrm{g}\left(\mathrm{Y}_{1}, \mathrm{Y}_{2}\right) \\
& -\omega_{1}\left(\mathrm{Y}_{1}\right) \omega_{1}\left(\mathrm{Y}_{2}\right) \\
& -\omega_{2}\left(\mathrm{Y}_{1}\right) \omega_{2}\left(\mathrm{Y}_{2}\right)
\end{aligned}
$$

where $\phi$ is $(1,1)$-tensor field on $\mathrm{N}$ (Bande and Hadjar, 2009). 
As mentioned above, we have two induced contact forms on the foliations $F_{1}$ and $F_{2}$. For to define almost contact structures on $F_{1}$ and $F_{2}$ we need the decomposability of $\phi$, i.e $\phi=\phi_{1}+\phi_{2}$. If $\phi \mathrm{TF}_{\mathrm{i}} \subset \mathrm{TF}_{\mathrm{i}}$ for $1 \leq \mathrm{i} \leq 2$, then $\phi$ is said to be decomposable. Thus, we have an almost contact structure $\left(\alpha_{1}, \mathrm{Z}_{1}, \phi_{1}\right) \quad\left(\operatorname{resp} .\left(\alpha_{2}, \mathrm{Z}_{2}, \phi_{2}\right)\right) \quad$ on $\mathrm{TF}_{2}\left(\right.$ resp. $\left.\mathrm{TF}_{1}\right)$ if $\phi$ is decomposable. Throughout the paper we assume that $\phi$ is decomposable. Moreover, we have

$$
\begin{aligned}
& \mathrm{g}\left(\mathrm{Y}_{1}, \mathrm{Z}_{\mathrm{i}}\right)=\alpha_{\mathrm{i}}\left(\mathrm{Y}_{1}\right), \quad \mathrm{g}\left(\mathrm{Z}_{\mathrm{i}}, \mathrm{Z}_{\mathrm{j}}\right)=\delta_{\mathrm{ij}}, \mathrm{g}(\mathrm{Z}, \mathrm{Z})=2, \\
& \nabla_{\mathrm{Z}_{\mathrm{i}}}^{\mathrm{Z}_{\mathrm{j}}}=0, \nabla_{\mathrm{Z}_{\mathrm{i}}}^{\phi}=0, \nabla_{\mathrm{Y}_{1}}^{\mathrm{Z}_{1}}=-\phi_{1} \mathrm{Y}_{1}, \nabla_{\mathrm{Y}_{1}}^{\mathrm{Z}_{2}}=-\phi_{2} \mathrm{Y}_{1}
\end{aligned}
$$

where $\phi=\phi_{1}+\phi_{2}$ and $\mathrm{Z}=\mathrm{Z}_{1}+\mathrm{Z}_{2}$.

The normality of an almost MCP manifold $\mathrm{N}$ has been given in (Bande and Hadjar, 2010). They defined two almost complex structures as,

$$
\begin{gathered}
\mathrm{J}=\phi-\omega_{2} \otimes \mathrm{Z}_{1}+\omega_{1} \otimes \mathrm{Z}_{2}, \\
\mathrm{~T}=\phi+\omega_{2} \otimes \mathrm{Z}_{1}-\omega_{1} \otimes \mathrm{Z}_{2} .
\end{gathered}
$$

$\mathrm{N}$ is said to be normal if $\mathrm{J}$ and $\mathrm{T}$ are integrable.

For $Y_{1}, Y_{2}$ horizontal vector fields we have the following curvature relations (Bande and Blair, 2013);

$$
\begin{aligned}
& \operatorname{Rm}\left(Y_{1}, Z\right) Y_{2}=g\left(Y_{1}, Y_{2}\right) Z \\
& \operatorname{Rm}\left(Y_{1}, Z\right) Z=-\phi^{2} Y_{1} \\
& \sigma\left(Y_{1}, Z\right)=0 \\
& \sigma(Z, Z)=2 p+2 q, \sigma\left(Z_{1}, Z_{1}\right)=2 p \\
& \sigma\left(Z_{2}, Z_{2}\right)=2 q, \sigma\left(Z_{1}, Z_{2}\right)=0
\end{aligned}
$$

where $\mathrm{Rm}$ is the Riemann curvature tensor and $\sigma$ is the Ricci curvature tensor of $\mathrm{N}$.

A NMCP manifold $\left(\mathrm{N}, \alpha_{1}, \alpha_{2}, \phi, \mathrm{Z}_{1}, \mathrm{Z}_{2}, \mathrm{~g}\right)$ is said to be a generalized quasi-Einstein (GQE) manifold if its Ricci tensor is not identically zero and satisfies;

$$
\begin{aligned}
\operatorname{Ric}\left(\mathrm{Y}_{1}, \mathrm{Y}_{2}\right) & =\lambda \mathrm{g}\left(\mathrm{Y}_{1}, \mathrm{Y}_{2}\right) \\
& +(2 \mathrm{p}-\lambda) \alpha_{1}\left(\mathrm{Y}_{1}\right) \alpha_{1}\left(\mathrm{Y}_{2}\right) \\
& +(2 \mathrm{q}-\lambda) \alpha_{2}\left(\mathrm{Y}_{1}\right) \alpha_{2}\left(\mathrm{Y}_{2}\right)
\end{aligned}
$$

for all vector fields $\mathrm{Y}_{1}, \mathrm{Y}_{2} \in \Gamma(\mathrm{TN})$ and $\lambda$ is non-zero scalar (Ünal, 2020).

\section{Lemma 1.}

A NMCP is a GQE manifold if and only if the $\sigma\left(\mathrm{Y}_{1}, \mathrm{Y}_{2}\right)=\lambda \mathrm{g}\left(\mathrm{Y}_{1}, \mathrm{Y}_{2}\right)$ for all $\mathrm{Y}_{1}, \mathrm{Y}_{2} \in \mathrm{H}$ (Ünal, 2020).

A Riemannian manifold $\mathrm{N}$ is called a space of generalized quasi-constant curvature if we have

$$
\begin{aligned}
& \operatorname{Rm}\left(Y_{1}, Y_{2}, Y_{3}, Y_{4}\right)=A\left[g\left(Y_{2}, Y_{3}\right) g\left(Y_{1}, Y_{4}\right)\right. \\
&\left.-g\left(Y_{1}, Y_{3}\right) g\left(Y_{2}, Y_{4}\right)\right] \\
&+\mathrm{B}\left[g\left(Y_{1}, Y_{4}\right) \eta_{1}\left(Y_{2}\right) \eta_{1}\left(Y_{3}\right)\right. \\
&-g\left(Y_{1}, Y_{3}\right) \eta_{1}\left(Y_{2}\right) \eta_{1}\left(Y_{4}\right) \\
&+ g\left(Y_{2}, Y_{3}\right) \eta_{1}\left(Y_{1}\right) \eta_{1}\left(Y_{4}\right) \\
&\left.-g\left(Y_{2}, Y_{4}\right) \eta_{1}\left(Y_{1}\right) \eta_{1}\left(Y_{3}\right)\right] \\
&+C\left[g\left(Y_{1}, Y_{4}\right) \eta_{2}\left(Y_{2}\right) \eta_{2}\left(Y_{3}\right)\right. \\
&-g\left(Y_{1}, Y_{3}\right) \eta_{2}\left(Y_{2}\right) \eta_{2}\left(Y_{4}\right) \\
&+g\left(X_{2}, X_{3}\right) \eta_{2}\left(X_{1}\right) \eta_{2}\left(X_{4}\right) \\
&\left.-g\left(Y_{2}, Y_{4}\right) \eta_{2}\left(Y_{1}\right) \eta_{2}\left(Y_{3}\right)\right]
\end{aligned}
$$

for all $Y_{1}, Y_{2}, Y_{3}, Y_{3} \in \Gamma(T N)(D e, 2004)$.

Presented author (Ünal, 2020) examined NMCP manifolds are space of generalized quasi-constant curvature and he proved that such manifolds are generalized quasi-Einstein.

\section{RESULTS AND DISCUSSION}

A NMCP manifold $\mathrm{N}$ is called by GQC-flat if $\mathrm{W}\left(\mathrm{Y}_{1}, \mathrm{Y}_{2}\right) \mathrm{Y}_{3}=0$ and also, $\mathrm{N}$ is said to be GQC-Zflat if $W\left(Y_{1}, Z\right) Y_{2}=0$ for all $Y_{1}, Y_{2}, Y_{3}$ vector fields on $\mathrm{N}$. In this section, we give many geometric properties of NMCP manifolds under the flatness conditions of GQC curvature tensor.

\section{Theorem 2.}

A GQC-Z-flat NMCP manifold is a GQE manifold. 


\section{Proof.}

Suppose that $\mathrm{N}$ satisfies for horizontal vector fields $Y_{1}, Y_{3}$. Then, from Equation (1) we get

$$
\begin{aligned}
0= & \operatorname{Rm}\left(\mathrm{Y}_{1}, \mathrm{Z}\right) \mathrm{Y}_{3}+\alpha\left[\sigma\left(\mathrm{Z}, \mathrm{Y}_{3}\right) \mathrm{Y}_{1}-\sigma\left(\mathrm{Y}_{1}, \mathrm{Y}_{3}\right) \mathrm{Z}\right] \\
& +\beta\left[\mathrm{g}\left(\mathrm{Z}, \mathrm{Y}_{3}\right) \mathrm{Q} \mathrm{Y}_{1}-\mathrm{g}\left(\mathrm{Y}_{1}, \mathrm{Y}_{3}\right) \mathrm{QZ}\right] \\
& -\frac{\gamma \mathrm{s}}{2 \mathrm{k}+1}\left(\frac{1}{2 \mathrm{k}}+\alpha+\beta\right)\left[\mathrm{g}\left(\mathrm{Z}, \mathrm{Y}_{3}\right) \mathrm{Y}_{1}-\mathrm{g}\left(\mathrm{Y}_{1}, \mathrm{Y}_{3}\right) \mathrm{Z}\right]
\end{aligned}
$$

By using Equation (3) and Equation (5) we get

$$
\begin{aligned}
0 & =g\left(Y_{1}, Y_{3}\right) Z-\alpha \sigma\left(Y_{1}, Y_{3}\right) Z \\
& -\beta g\left(Y_{1}, Y_{3}\right) Q Z \\
& +\frac{\gamma s}{2 k+1}\left(\frac{1}{2 k}+\alpha+\beta\right) g\left(Y_{1}, Y_{3}\right) Z
\end{aligned}
$$

and taking inner product with $\mathrm{Z}$, we obtain

$$
\begin{aligned}
\sigma\left(\mathrm{Y}_{1}, \mathrm{Y}_{3}\right) & =\frac{1}{\alpha}(1-2 \mathrm{~m} \beta \\
& \left.+\frac{\gamma \mathrm{s}}{2 \mathrm{k}+1}\left(\frac{1}{2 \mathrm{k}}+\alpha+\beta\right)\right) \mathrm{g}\left(\mathrm{Y}_{1}, \mathrm{Y}_{3}\right) .
\end{aligned}
$$

Therefore, by consider the Lemma 1 . we get

$$
\begin{aligned}
\sigma\left(\mathrm{Y}_{1}, \mathrm{Y}_{3}\right) & =\frac{1}{\alpha}(1-2 \mathrm{~m} \beta \\
& \left.+\frac{\gamma \mathrm{s}}{2 \mathrm{k}+1}\left(\frac{1}{2 \mathrm{k}}+\alpha+\beta\right)\right) \mathrm{g}\left(\mathrm{Y}_{1}, \mathrm{Y}_{3}\right) \\
& +\left(2 \mathrm{~m}-\frac{1}{\alpha}(1-2 \mathrm{~m} \beta\right. \\
& \left.\left.+\frac{\gamma \mathrm{s}}{2 \mathrm{k}+1}\left(\frac{1}{2 \mathrm{k}}+\alpha+\beta\right)\right)\right) \eta_{1}\left(\mathrm{Y}_{1}\right) \eta_{1}\left(\mathrm{Y}_{3}\right) \\
& +\left(2 \mathrm{n}-\frac{1}{\alpha}(1-2 \mathrm{~m} \beta\right. \\
& \left.\left.+\frac{\gamma \mathrm{s}}{2 \mathrm{k}+1}\left(\frac{1}{2 \mathrm{k}}+\alpha+\beta\right)\right)\right) \eta_{2}\left(\mathrm{Y}_{1}\right) \eta_{2}\left(\mathrm{Y}_{3}\right)
\end{aligned}
$$

which provides $\mathrm{N}$ is a GQE manifold.

\section{Theorem 3.}

Let $\mathrm{N}$ be an Einstein NMCP manifold. If $\mathrm{N}$ is also GQC-flat, then $\mathrm{N}$ is a generalized real space form.

\section{Proof.}

Suppose that N is a GQC-flat NMCP manifold. Then from Equation (1), we get

$$
\begin{aligned}
\operatorname{Rm}\left(\mathrm{Y}_{1}, \mathrm{Y}_{2}\right) \mathrm{Y}_{3}=-\alpha\left[\sigma\left(\mathrm{Y}_{2}, \mathrm{Y}_{3}\right) \mathrm{Y}_{1}\right. \\
\left.-\sigma\left(\mathrm{Y}_{1}, \mathrm{Y}_{3}\right) \mathrm{Y}_{2}\right] \\
-\beta\left[\mathrm{g}\left(\mathrm{Y}_{2}, \mathrm{Y}_{3}\right) \mathrm{Q} \mathrm{Y}_{1}-\mathrm{g}\left(\mathrm{Y}_{1}, \mathrm{Y}_{3}\right) \mathrm{Q} \mathrm{Y}_{2}\right] \\
+\frac{\gamma \mathrm{s}}{2 \mathrm{k}+1}\left(\frac{1}{2 \mathrm{k}}+\alpha+\beta\right)\left[\mathrm{g}\left(\mathrm{Y}_{2}, \mathrm{Y}_{3}\right) \mathrm{Y}_{1}\right. \\
\left.-\mathrm{g}\left(\mathrm{Y}_{1}, \mathrm{Y}_{3}\right) \mathrm{Y}_{2}\right]
\end{aligned}
$$

and thus, we have

$$
\begin{aligned}
\operatorname{Rm}\left(Y_{1}, Y_{2}, Y_{3}, Y_{4}\right) & =-\alpha\left[\sigma\left(Y_{2}, Y_{3}\right) g\left(Y_{1}, Y_{4}\right)\right. \\
& \left.-\sigma\left(Y_{1}, Y_{3}\right) g\left(Y_{2}, Y_{4}\right)\right] \\
& -\beta\left[g\left(Y_{2}, Y_{3}\right) \sigma\left(Y_{1}, Y_{4}\right)\right. \\
& \left.-g\left(Y_{1}, Y_{3}\right) \sigma\left(Y_{2}, Y_{4}\right)\right] \\
& +\frac{\gamma s}{2 k+1}\left(\frac{1}{2 k}+\alpha\right. \\
& +\beta)\left[g\left(Y_{2}, Y_{3}\right) g\left(Y_{1}, Y_{4}\right)\right. \\
& \left.-g\left(Y_{1}, Y_{3}\right) g\left(Y_{2}, Y_{4}\right)\right]
\end{aligned}
$$

for all $\mathrm{Y}_{1}, \mathrm{Y}_{2}, \mathrm{Y}_{3}, \mathrm{Y}_{4} \in \Gamma(\mathrm{TN})$. If $\mathrm{N}$ is an Einstein manifold with non-zero cosmology constant $\lambda$, then we get

$$
\begin{aligned}
\operatorname{Rm}\left(Y_{1}, Y_{2}, Y_{3}, Y_{4}\right) & =F\left[g\left(Y_{2}, Y_{3}\right) g\left(Y_{1}, Y_{4}\right)\right. \\
& \left.-g\left(Y_{1}, Y_{3}\right) g\left(Y_{2}, Y_{4}\right)\right]
\end{aligned}
$$

where $F=\left(-\alpha-\beta+\frac{\gamma \mathrm{s}}{2 \mathrm{k}+1}\left(\frac{1}{2 \mathrm{k}}+\alpha+\beta\right)\right) \lambda$. This shows us $\mathrm{N}$ is a space form.

\section{Theorem 4.}

Let $\mathrm{N}$ be a GQE NMCP manifold. If $\mathrm{N}$ is GQCflat then it is a space of generalized quasi-constant curvature.

\section{Proof.}

Let $\mathrm{N}$ be a GQC-flat GQE NMCP manifold. Then from Equation (1) and Equation (7), we obtain $\mathrm{A}=-\alpha-\beta+\frac{\gamma \mathrm{s}}{2 \mathrm{k}+1}\left(\frac{1}{2 \mathrm{k}}+\alpha+\beta\right)$ 


$$
\begin{aligned}
& B=(-\alpha-\beta)(2 m-\lambda) \\
& C=(-\alpha-\beta)(2 n-\lambda)
\end{aligned}
$$

So, $\mathrm{N}$ is a space of generalized quasi-constant curvature.

\section{Theorem 5.}

\section{Proof.}

A GQC-flat NMCP manifold is a GQE manifold.

Suppose that N is a GQC-flat NMCP manifold. Let take an orthonormal basis of $\mathrm{N}$ as

$\left\{E_{1}, E_{2}, \ldots, E_{2 m}, E_{2 m+1}, \ldots, E_{2 n}, Z_{1}, Z_{2}\right\}$.

By setting $\quad \mathrm{Y}_{2}=\mathrm{Y}_{3}=\mathrm{E}_{\mathrm{i}} \quad, \quad 1 \leq \mathrm{i} \leq \mathrm{k}-2$, $k=2 m+2 n+2$ and taking sum over $i$, we get

$$
\begin{aligned}
\sum_{\mathrm{i}=1}^{\mathrm{k}-2} \mathrm{Rm}\left(\mathrm{Y}_{1}, \mathrm{E}_{\mathrm{i}}, \mathrm{E}_{\mathrm{i}}, \mathrm{Y}_{4}\right) & =\sum_{\mathrm{i}=1}^{\mathrm{k}-2}\left\{-\alpha\left[\sigma\left(\mathrm{E}_{\mathrm{i}}, \mathrm{E}_{\mathrm{i}}\right) \mathrm{g}\left(\mathrm{Y}_{1}, \mathrm{Y}_{4}\right)\right.\right. \\
& \left.-\sigma\left(\mathrm{Y}_{1}, \mathrm{E}_{\mathrm{i}}\right) \mathrm{g}\left(\mathrm{E}_{\mathrm{i}}, \mathrm{Y}_{4}\right)\right] \\
& -\beta\left[\mathrm{g}\left(\mathrm{E}_{\mathrm{i}}, \mathrm{E}_{\mathrm{i}}\right) \sigma\left(\mathrm{Y}_{1}, \mathrm{Y}_{4}\right)\right. \\
& \left.-\mathrm{g}\left(\mathrm{Y}_{1}, \mathrm{E}_{\mathrm{i}}\right) \sigma\left(\mathrm{E}_{\mathrm{i}}, \mathrm{Y}_{4}\right)\right] \\
& +\frac{\gamma \mathrm{s}}{2 \mathrm{k}+1}\left(\frac{1}{2 \mathrm{k}}+\alpha\right. \\
& +\beta)\left[\mathrm{g}\left(\mathrm{E}_{\mathrm{i}}, \mathrm{E}_{\mathrm{i}}\right) \mathrm{g}\left(\mathrm{Y}_{1}, \mathrm{Y}_{4}\right)\right. \\
& \left.\left.-\mathrm{g}\left(\mathrm{Y}_{1}, \mathrm{E}_{\mathrm{i}}\right) \mathrm{g}\left(\mathrm{E}_{\mathrm{i}}, \mathrm{Y}_{4}\right)\right]\right\}
\end{aligned}
$$

for horizontal vector fields $\mathrm{Y}_{1}, \mathrm{Y}_{4}$. On the hand since we have

$$
\begin{aligned}
& \sum_{i=1}^{\mathrm{k}-2} \sigma\left(\mathrm{E}_{\mathrm{i}}, \mathrm{E}_{\mathrm{i}}\right)=\mathrm{s}-2 \mathrm{~m}-2 \mathrm{n}, \\
& \sum_{\mathrm{i}=1}^{\mathrm{k}-2} \sigma\left(\mathrm{Y}_{1}, \mathrm{E}_{\mathrm{i}}\right) \mathrm{g}\left(\mathrm{E}_{\mathrm{i}}, \mathrm{Y}_{4}\right)=\sigma\left(\mathrm{Y}_{1}, \mathrm{Y}_{4}\right), \\
& \sum_{\mathrm{i}=1}^{\mathrm{k}-2} \mathrm{~g}\left(\mathrm{Y}_{1}, \mathrm{E}_{\mathrm{i}}\right) \mathrm{g}\left(\mathrm{E}_{\mathrm{i}}, \mathrm{Y}_{4}\right)=\mathrm{g}\left(\mathrm{Y}_{1}, \mathrm{Y}_{4}\right)
\end{aligned}
$$

then, we obtain

$$
\begin{aligned}
\sigma\left(\mathrm{Y}_{1}, \mathrm{Y}_{4}\right)= & -\alpha\left((\mathrm{s}-(\mathrm{k}-2)) \mathrm{g}\left(\mathrm{Y}_{1}, \mathrm{Y}_{4}\right)-\sigma\left(\mathrm{Y}_{1}, \mathrm{Y}_{4}\right)\right) \\
& -\beta\left((\mathrm{k}-2) \sigma\left(\mathrm{Y}_{1}, \mathrm{Y}_{4}\right)-\mathrm{g}\left(\mathrm{Y}_{1}, \mathrm{Y}_{4}\right)\right) \\
& +\frac{\gamma \mathrm{s}}{2 \mathrm{k}+1}\left(\frac{1}{2 \mathrm{k}}+\alpha+\beta\right)\left((\mathrm{k}-2) \mathrm{g}\left(\mathrm{Y}_{1}, \mathrm{Y}_{4}\right)\right. \\
& \left.-\mathrm{g}\left(\mathrm{Y}_{1}, \mathrm{Y}_{4}\right)\right) .
\end{aligned}
$$

Finally, we get

$$
\sigma\left(Y_{1}, Y_{4}\right)=\lambda g\left(Y_{1}, Y_{4}\right)
$$

where

$$
\begin{aligned}
\lambda= & \frac{1}{1-\alpha+\beta(\mathrm{k}-2)}(-\alpha(\mathrm{s}-(\mathrm{k}-2)) \\
& \left.+\beta+\frac{\gamma \mathrm{s}}{2 \mathrm{k}+1}\left(\frac{1}{2 \mathrm{k}}+\alpha+\beta\right)(\mathrm{k}-3)\right) .
\end{aligned}
$$

Thus from Lemma $1 . \mathrm{N}$ is a GQE manifold.

The sectional curvature of a Riemannian manifold gives us a significant geometric interpretation. In the contact geometry, we have $\phi$ sectional curvature and $\xi$-sectional curvature. Similarly, we have $\phi$-sectional curvature and $\mathrm{Z}-$ sectional curvature for NMCP manifolds.

\section{Corollary 6.}

The sectional curvature of a GQC-flat NMCP manifold is given by

$$
\begin{aligned}
\sec (\mathrm{X}, \mathrm{Y})= & -\frac{\alpha+\beta}{1-\alpha+\beta(\mathrm{k}-2)}(-\alpha(\mathrm{s}-(\mathrm{k}-2)) \\
& \left.+\beta+\frac{\gamma \mathrm{s}}{2 \mathrm{k}+1}\left(\frac{1}{2 \mathrm{k}}+\alpha+\beta\right)(\mathrm{k}-3)\right) \\
& +\frac{\gamma \mathrm{s}}{2 \mathrm{k}+1}\left(\frac{1}{2 \mathrm{k}}+\alpha+\beta\right)
\end{aligned}
$$

for unit and mutually orthogonal horizontal vector fields $\mathrm{X}, \mathrm{Y}$.

Proof.

Let $\mathrm{N}$ be a GQC-flat NMCP manifold. By taking $\mathrm{Y}_{1}=\mathrm{Y}_{4}=\mathrm{X}, \mathrm{Y}_{2}=\mathrm{Y}_{3}=\mathrm{Y}$ for unit and mutually orthogonal horizontal vector fields $\mathrm{X}, \mathrm{Y}$, we have

$$
\begin{aligned}
\operatorname{Rm}(X, Y, Y, X)= & -\frac{\alpha+\beta}{1-\alpha+\beta(\mathrm{k}-2)}(-\alpha(\mathrm{s}-(\mathrm{k}-2)) \\
& \left.+\beta+\frac{\gamma \mathrm{s}}{2 \mathrm{k}+1}\left(\frac{1}{2 \mathrm{k}}+\alpha+\beta\right)(\mathrm{k}-3)\right) \\
& +\frac{\gamma \mathrm{s}}{2 \mathrm{k}+1}\left(\frac{1}{2 \mathrm{k}}+\alpha+\beta\right) .
\end{aligned}
$$

Thus the proof is desired. 


\section{Corollary 7.}

Let $\mathrm{N}$ be a GQC-flat NMCP manifold. If the scalar curvature is constant, then $\mathrm{N}$ has constant sectional curvature.

\section{CONCLUSION}

In the theory of relativity, solutions of the Einstein field equation are a major notion. Since the Riemannian geometry has many applications in the general relativity, this subject is also interesting for differential geometers. In differential geometry of manifolds, researchers examined some special manifolds as the solitons of the Einstein field equation. One of these manifolds is GQE manifolds. In (Ünal, 2020) we defined GQE NMCP manifold and thus we present the applications of GQE manifolds in contact geometry. One can see that NMCP manifolds could be special solitons of Einstein fields equations. Considering all these important details, we focus on the NMCP manifolds with a general curvature tensor. We present some related results to general relativity via GQC curvature tensor. We hope the presented paper will be a nice reference for future works.

\section{CONFLICT OF INTEREST STATEMENT}

The author declares that there is no conflict of interest in this study.

\section{RESEARCH AND PUBLICATION ETHICS STATEMENT}

The author declares that research and publication ethics are followed in this study.

\section{REFERENCES}

Acet, B.E., 2018. A note on Ricci solitons on paraSasakian manifolds. Erzincan Üniversitesi Fen Bilimleri Enstitüsü Dergisi, 11(2):237-242.

Baishya, K.K., Chowdhury, P.R., 2016. On generalized quasi-conformal $\mathrm{N}(\kappa, \mu)-\quad$ manifolds.
Communications of the Korean Mathematical Society, 31(1):163-176.

Baishya, K.K., Chowdhury, P.R., 2017 Kenmotsu manifolds equipped with generalized quasiconformal curvature tensor. Global Journal of Pure and Applied Mathematics, 13(6):2493-2502.

Baishya, K.K., Chowdhury, P.R., 2017 Semi-symmetry type LP-Sasakian manifolds. Acta Mathematica Academiae Paedagogicae Nyregyhaziensis, 33:6783.

Baishya, K. K., 2017. Ricci solitons in Sasakian manifold. Afrika Matematika, 28: 1061-1066.

Bande, G., Hadjar, A., 2005. Contact pairs. Tohoku Mathematical Journal, Second Series, 57(2):247260.

Bande, G., Hadjar, A., 2009. Contact pair structures and associated metrics. In Differential Geometry, 1(1):266-275

Bande, G., Hadjar, A., 2010. On normal contact pairs. International Journal of Mathematics, 21(06):737754.

Bande, G., Blair, D.E., Hadjar, A., 2013. On the curvature of metric contact pairs. Mediterranean journal of mathematics, 10(2): 989-1009.

Bande, G., Blair, D.E., 2013. Symmetry in the geometry of metric contact pairs. Mathematische Nachrichten, 286:1701-1709

Bande, G., Blair, D. E., Hadjar, A., 2015. Bochner and conformal flatness of normal metric contact pairs, Annals of Global Analysis and Geometry, 48(1):4756.

Blair, D.E., Ludden, G.D., Yano, K., 1974. Geometry of complex manifolds similar to the Calabi-Eckmann manifolds. Journal of Differential Geometry, 9(2):263-274.

De, U. C., Ghosh, G. C., 2004. On generalized quasiEinstein manifolds, Kyungpook Mathematical Journal, 44:607-615.

Ünal, ì., 2020. Some flatness conditions on normal metric contact pairs. Communications Faculty of Sciences University of Ankara Series A1 Mathematics and Statistics, 69(2):262-271.

Ünal, İ., 2020. Generalized Quasi-Einstein manifolds in contact geometry. Mathematics, 8(9):1592.

Ünal, İ., 2021. On metric contact pairs with certain semisymmetry conditions. Politeknik Dergisi, 1- 